\title{
3 Research Square

\section{Adrenal Stereotactic Body Radiation Therapy: The Effects of a Full and Empty Stomach on Radiation Dose to Organs at Risk}

Osamu Tanaka ( $\nabla$ c.bluered@gmail.com )

Gifu Municipal Hospital https://orcid.org/0000-0002-7189-8407

Takuya Taniguchi

Asahi University Hspital

Kousei Ono

Asahi University Hospital

Shuto Nakaya

Asahi University Hospital

Takuji Kiryu

Asahi University Hospital

Chiyoko Makita

Gifu University Hospital

Masayuki Matsuo

Gifu University Hospital

\section{Research Article}

Keywords: radiotherapy, stereotactic body radiotherapy, adrenal tumor, Organ at Risk, Stomach

Posted Date: April 19th, 2021

DOI: https://doi.org/10.21203/rs.3.rs-312582/v2

License: (c) (i) This work is licensed under a Creative Commons Attribution 4.0 International License.

Read Full License

Version of Record: A version of this preprint was published at International Journal of Radiation Oncology*Biology*Physics on November 1st, 2021. See the published version at https://doi.org/10.1016/j.jijrobp.2021.07.1333. 


\section{Abstract}

\section{Background}

Stereotactic Body Radiation Therapy (SBRT) has been reported to be curative in the treatment of oligometastases to the adrenal glands. However, the adrenals are surrounded by radiation-sensitive organs. We performed an Organ at Risk (OAR) analysis for SBRT to the left adrenal gland based on gastric state.

\section{Patients and Methods}

Twenty random stomachs were divided into "empty" or "full" groups of 10 each based on size. The PTV dose was $54 \mathrm{~Gy} / 6 \mathrm{fx}$ and D 95 coverage of PTV(CTV) (the dose to $95 \%$ of the PTV volume).

\section{Results}

The gastric OAR dose in the empty group was significantly lower than the full group. The OAR dose to the left kidney in the empty group was significantly higher than in the full group.

\section{Conclusion}

The smaller the stomach size, the lower the dose to the stomach. It is therefore better to perform SBRT on patients with an empty stomach.

\section{Introduction}

Evidence of the effectiveness of Stereotactic Body Radiation Therapy (SBRT) has recently been published [1-11]. Moreover, it has been reported that curative treatment for oligometastatic cancer where the primary lesion is controlled improves the disease-free recurrence time and overall survival. The adrenal glands are often the site of a solitary thoracic, abdominal, or pelvic metastasis, especially from lung cancer [3-11].

There is also a form of adrenal recurrence after radical resection of the primary lesion. There are reports that there is an advantage to use SBRT when there is an adrenal metastasis at the time of lung or breast cancer diagnosis, or when an adrenal metastasis appears after the completion of primary tumor treatment.

However, the adrenal glands are surrounded by radiation-sensitive organs, such as the pancreas, kidneys, small intestine, biliary system, and stomach. Thus, treatment planning requires close regulation due to the dose limit to the OAR. Radiation therapy can be adjusted for gastric capacity and cholecystic capacity (both include dietary restrictions). Hence we performed an OAR (Intestine, Pancreas, Liver, Right kidney, and spinal cord) analysis of SBRT to the left adrenal gland based on gastric state (empty or full stomach) at the time of irradiation. We examined whether it was possible to reduce the dose to the OAR. 


\section{Materials \& Methods}

Included patients had prostate cancer or uterine cancer with treatment planning CTs images from the diaphragm to the pelvis and no history of abdominal organ dysfunction (ie. cirrhosis, pancreatitis, renal atrophy). Of the 20 stomachs, that met our inclusion criteria, we divided 10 into a median larger group (considered a full stomach) and the other 10 into a smaller group (considered an empty stomach).

Deep inspiration breath hold (DIBH) technique was used with the Abches system [12]. Patients were immobilized using an alpha cradle in the supine position with both arms over their heads. The main component of Abches was set over the iliac crest with two fulcrums placed and marked at the patient's sternum and abdomen. The Abches system was made from resin without electronic parts; thus, they only had minimal influence on dose calculation.

The "empty group" was defined as the smaller stomach group $(n=10)$, whereas the "full group" was the larger stomach group $(n=10)$. All radiation plans were created with a 3D treatment planning system (Elekta's $\mathrm{XiO}{ }^{\circledR}$ treatment planning system and focal contouring system, Hamburg, Germany). An Elekta Synergy linear accelerator with $6 \mathrm{MV}$ photon energy was used.

Adrenal ground and OAR were outlined by an abdominal radiation oncologist and medical physicists created the beam plans and the same radiation oncologist evaluated them. The radiation therapist performing the contouring and the medical physicist performing the treatment plan were blinded to patient information. the CTV was equal to the GTV. A $3 \mathrm{~mm}$ isotropic margin was used to obtain the corresponding PTV. The prescribed PTV dose was $54 \mathrm{~Gy} / 6 \mathrm{fx}$ and D 95 coverage of PTV (the dose to $95 \%$ of the PTV volume). OAR dose limits are shown in Table 1. The capacity of the stomach was measured for its effects on OAR. Treatment regimens were analyzed with a dose volume histogram, and a one-way analysis of variance PTV was performed. All data are presented as mean \pm standard deviation. We defined $\mathrm{P}<0.05$ as statistically significant. We used a two-sided Wilcoxon rank-sum/Mann-Whitney $U$ test using the Excel statistical software package (Excel-statistics 2015; Social Survey Research Information Co., Ltd., Tokyo, Japan). The primary results (D/V parameters) should be reported with both measures of central tendency (mean) and spread (SD or $95 \% \mathrm{Cl}$ ). The very small sample size would indicate that the spread of the measurements would have had to have been extremely small $(S D<2)$ for the results to have been statistically significant. We should report of the full results, i.e., a table of the PTV and OAR sizes as well as dosimetric parameters of each individual patient, which would be beneficial in assessing the results.

However, the internal organs to OAR this time, there are several variations in the case, depending on the shape of the parenchymal organs (liver, pancreas, kidney) and the distance to the stomach. The amount and spread of visceral fat also vary extensively. Therefore, we have omitted the SD to improve the readability.

This study is a retrospective cohort comparison study. Informed consent was waived, and an opt out was available on the hospital homepage. 


\section{Results}

The stomach size of the empty group was $226 \pm 98 \mathrm{~cm}^{3}$, and the size of the full group was $480 \pm 91 \mathrm{~cm}^{3}$. A sample image of SBRT is shown in Figure 1. Interesting information can be observed in the tables. For example, readers want to know whether the stomach is empty do the other OARs receive higher dose (answer may be yes for left kidney).

The relationship between stomach size and OAR dose was measured. The gastric OAR dose in the empty group was significantly lower than in the full group (D5 and D10) (Table.2). However, the OAR dose to the left kidney in the empty group was statistically significantly higher than in the full group (V12, V15, and V21). From table 2, it is evident that with an empty stomach, the left kidney receives significantly higher dose; this is of outmost importance because radiation oncologist must make a decision, i.e., should physician prefer stomach of kidney preservation. In this way, stomach seems to act as a natural spacer for abdominal organs.

All other organs (intestines, pancreas, liver, right kidney, and spinal cord) had equivocal OAR doses between the two groups.

\section{Discussion}

According to previous reports[13, 14], which is the most suitable balance between protecting the stomach and kidney? How should I simulate a patient to assure the best dosimetrical outcome?

It was found that a smaller stomach was better separated from the left adrenal gland, and therefore received a smaller radiation dose. The pancreas is between the left adrenal gland and the stomach. Both the pancreas and the adrenal glands are retroperitoneal organs, but the stomach is not. In addition, although the image was taken in the supine position this time, it is possible that the distance between the stomach and the left adrenal gland can be further increased by positioning the patient in the left lateral decubitus or prone position.

In contrast to the stomach, the kidney is in contact with the adrenal gland as a retroperitoneal organ, and it is difficult to control its movement. However, it was observed that the larger the stomach, the lower the dose to the left kidney. The reason for this could be that the stomach squeezes the pancreas and the kidneys dorsally, causing the kidneys to move caudally and have a reduced radiation exposure. Moreover, it is important for patients with impaired renal function to increase gastric distension and move the left kidney caudally to reduce the dose to the kidney. in contrast, if the stomach has a lesion, the stomach and the adrenal glands may be separated from each other on an empty stomach. In addition, because the stomach on an empty stomach tends to maintain reproducibility [Tanaka BJR], it may be possible to treat with the same accuracy as MR-liniac if the treatment is performed with high reproducibility on an empty stomach [15-16]. 
It has also been shown gastric volume varies significantly both between and within fractions (inter- and intra-fraction motion)[13-16]. Because of close proximity of the stomach, patients with left-sided tumors were advised to fast for at least $2.5 \mathrm{~h}$ before simulation and treatment. Their results using MR-guided SBRT showed that, for left-sided adrenal tumors, the stomach V15 significantly decreased post-treatment; from a mean of $29.6 \mathrm{cc}$ (5th-95th percentile range: 6.3-72.4 cc) pre-treatment, to a post-treatment mean volume of $24.9 \mathrm{cc}(3.9-55.2 \mathrm{cc})(\mathrm{p}<0.0003)$.

In addition, we thought it was necessary to irradiate the stomach with the same shape each time. As a method of making the shape of the stomach the same at each irradiation, compare whether it is better to make the stomach empty, or to have the patient drink about $400 \mathrm{ml}$ of water each time before the treatment plan and irradiation to achieve reproducibility as a full stomach. did.

Control of bladder volume by drinking water is well known in IMRT for prostate cancer. However, it is done on the premise that the morphology of the bladder does not change so much as the capacity of the bladder increases. However, the stomach is a very moving organ.

Therefore, we thought that it would be difficult to match the shape by drinking water each time, and considered the empty stomach due to fasting as the control group. It was reported that the morphology of the stomach can be maintained by fasting the stomach (Tanaka). However, if the empty stomach causes an increase in the dose to the stomach, it is a complete fall, and this test was conducted.

It is difficult to reliably measure the small intestine because it is in a different position every day. In fact, even with a cone beam CT the position of the small intestine changes every time. It is true that drinking water when treating the stomach may change the dilation of the duodenum, but this degree of dilation varies from day to day, so it is difficult to control the small intestine.

The dose to the pancreas was not affected by stomach morphology. This is considered to be due to the close proximity of the left adrenal gland and the pancreas. However, the size of the pancreas also varies greatly for each patient, and visceral fat may easily adhere to the area around the pancreas, which may also be reduced by gaining weight.

\section{Clinical practice}

An empty and full stomach had an opposite effect on OAR to the stomach and left kidney during adrenal irradiation. Therefore, it is necessary to choose whether to prepare for this by reducing the dose to the stomach or reducing the dose to the left kidney. Findings here also suggest that it is useful to obtain treatment planning CTs with an empty and full stomach, permitting the creation of two SBRT plans.

In terms of choosing between SBRT with an empty or full, it is appropriate to decide this based on renal function and gastrointestinal condition (gastritis, etc.). Adrenal tumors are more likely to be metastatic deposits compared with primary tumors. Chemotherapy is often used for metastases, so it would be desirable to reduce the radiation dose to the kidneys in such cases. 
It is also necessary to consider the positional relationship of organs, especially given differences in visceral fat between patients. The distance between the organ and the adrenal gland was not taken into consideration in this work, but it is expected that the more visceral fat, the greater the distance between the adrenal gland and the irradiated organ. If increasing the amount of visceral fat reduces the dose to OAR, temporarily gaining weight may be a solution to this issue.

\section{Limitations}

The following three issues can be considered for improvements in future works. 1) SBRT to the right adrenal gland. Unlike the left adrenal gland, the right adrenal gland has less OAR but might involve an increased radiation dose to the inferior vena cava and biliary/bile duct pancreatic duct. Verification of this is required. 2) Decrease the radiation dose to the target due to increased OAR restrictions as the dose increases. In general, the higher the dose, the higher the cure rate (SBRT to the lungs and liver in particular). Therefore, it is important to find a dose that can be traded off $[6,7]$. 3) It is desirable to place a gold marker in order to improve accuracy, but there is a risk of inserting it percutaneously. Because the adrenal gland is an organ that cannot be seen on X-ray, reproducibility is usually attempted by aligning it with the position of the vertebral body. However, it is also necessary to consider a method for narrowing the PTV margin by placing a gold marker.

\section{Future Outlook (compared with MR-Linac)}

It is expected that the number of indications for SBRT will continue to increase in the future. It is necessary to establish a plan to protect OAR by administering the minimum prescribed dose. Techniques for artificially moving the position of the OAR (sometimes PTV) to remove the heart from the irradiation field, such as respiratory synchronization that is used in breast conserving therapy in the management of left breast cancer will continue to be required. It is true that the shape of the body surface and the appearance of MR-Linac have made it possible to understand information on the body's surface and well within the abdomen.

This study reveals that the adrenal glands may be able to regulate the radiation dose to the stomach and left kidney by changing the shape (size) of the stomach. MRI-Linac can reduce the target's intra- and intervariance but not the contents of the stomach [17]. Therefore, we believe that MR-Linac will become widespread, but also that such a method of artificially changing the shape and position of organs is necessary.

Therefore, if the organ position can be controlled by some kind of pretreatment, it is worth attempting.

\section{Conclusion}

When SBRT was performed on the left adrenal gland, it was found that the smaller the stomach size, the lower the dose to the stomach. The stomach is an abdominal organ that can be artificially resized. Therefore, it is better to perform SBRT on patients with an empty stomach. Furthermore, because it is 
easy for the empty stomach to have the same stomach shape every time due to fasting, it is better to plan SBRT on an empty stomach than to plan with the full stomach.

\section{Declarations}

Ethical Approval and Consent to participate

National Clinical Trial number UMIN000043471

Consent for publication

All authors approved

Availability of supporting data

Available

Competing interests

None

Funding

None

\section{Authors' contributions}

Conception and design of the study: Tanaka, Taniguchi, Ono

Analysis and interpretation of data: Nakaya

Collection and assembly of data: Nakaya

Drafting of the article: Tanaka, Kiryu, Makita

Critical revision of the article for important intellectual content: Tanaka

Final approval of the article: Matsuo

\section{Acknowledgements}

None

\section{Conflict of interest}

There are no conflicts of interest to report. 


\section{Financial support}

None

\section{Data sharing}

None

\section{References}

1) Helis $C A$, Hughes RT, Nieto $K$, Ufondu A, Daugherty EC, Farris MK.. Adrenal SBRT: a multiinstitutional review of treatment outcomes and toxicity. Clin Exp Metastasis. 37(5):585-592, 2020 doi: $10.1007 / \mathrm{s} 10585-020-10052-0$

2) Chen WC, Baal JD, Baal U, Pai J, Gottschalk A, Boreta L, Braunstein SE, Raleigh DR. Stereotactic body radiation therapy of adrenal metastases: a pooled meta-analysis and systematic review of 39 studies with 1006 patients. Int J Radiat Oncol Biol Phys. 107(1):48-61, 2020. doi:

10.1016/j.ijrobp.2020.01.017

3) Rudra S, Malik R, Ranck MC, Farrey K, Golden DW, Hasselle MD, Weichselbaum RR, Salama JK. Stereotactic body radiation therapy for curative treatment of adrenal metastases. Technol Cancer Res Treat.12(3):217-224, 2013 doi: 10.7785/tcrt.2012.500320

4) Malone J, Pantarotto JR, Tiberi $D$, Malone S. Adrenal oligometastasis cured with stereotactic ablative radiotherapy. Radiol Case Rep. 15(11):2266-2270, 2020 doi: 10.1016/j.radcr.2020.08.056

5) König L, Häfner MF, Katayama S, oerber SA, Tonndorf-Martini E, Bernhardt D, von Nettelbladt B, Weykamp F, Hoegen P, Klüter S, Susko MS, Debus J, Hörner-Rieber J. Stereotactic body radiotherapy (SBRT) for adrenal metastases of oligometastatic or oligoprogressive tumor patients. Radiat Oncol. 15(1):30, 2020 doi: 10.1186/s13014-020-1480-0

6) Plichta K, Camden N, Furqan M, Hejleh TA, Clamon GH, Zhang J, Flynn RT, Bhatia SK, Smith MC, Buatti JM, Allen BG. SBRT to adrenal metastases provides high local control with minimal toxicity. Adv Radiat Oncol. 2(4):581-587, 2017 doi: 10.1016/j.adro.2017.07.011

7) Figura NB, Oliver DE, Mohammadi H, Martinez K, Grass GD, Hoffe SE, Johnstone PAS, Frakes JM. Novel dose escalation approaches for stereotactic body radiotherapy to adrenal oligometastases: a single-institution experience. Am J Clin Oncol. 43(2):107-114, 2020

8) Voglhuber T, Kessel KA, Oechsner M, Vogel MME, Gschwend JE, Combs SE. Single-institutional outcome-analysis of low-dose stereotactic body radiation therapy (SBRT) of adrenal gland metastases. BMC Cancer. 20(1):536, 2020 doi: 10.1186/s12885-020-07030-w 
9) Buergy D, Rabe L, Siebenlist K, Stieler F, Fleckenstein J, Giordano FA, Wenz F, Boda-Heggemann J. Treatment of adrenal metastases with conventional or hypofractionated image-guided radiation therapy patterns and outcomes. J Anticancer Res. 38(8):4789-4796, 2018 doi: 10.21873/anticanres.12788

10) Sonier M, Chu W, Lalani N, Erler D, Cheung P, Korol R. Implementation of a volumetric modulated arc therapy treatment planning solution for kidney and adrenal stereotactic body radiation therapy. Med Dosim. 41(4):323-328, 2016 doi: 10.1016/j.meddos.2016.09.001

11) Arcidiacono F, Aristei $C$, Marchionni A, Italiani M, Fulcheri CPL, Saldi S, Casale M, Ingrosso G, Anselmo P, Maranzano E. Stereotactic body radiotherapy for adrenal oligometastasis in lung cancer patients. Br J Radiol. 93(1115):20200645, 2020 doi: 10.1259/bjr.20200645

12) Melissa de Kuijer, Jaap van Egmond, E Kouwenhoven, Dieke Bruijn-Krist , Heleen Ceha , Mirjam Mast. Breath-hold versus mid-ventilation in SBRT of adrenal metastases. Tech Innov Patient Support Radiat Oncol. 16;12:23-27, 2019 doi: 10.1016/j.tipsro.2019.11.007.

13) McPartlin AJ, Li XA, Kershaw LE Heide U, Kerkmeijer L, Lawton C, Mahmood U, Pos F, van As N, van Herk M, Vesprini D, van der Voort van Zyp J, Tree A, Choudhury A; MR-Linac consortium. MRI-guided prostate adaptive radiotherapy - A systematic review, Radiother Oncol. 119(3), 371-80, 2016. DOI: 10.1016/j.radonc.2016.04.014

14) Cao Y, Tseng CL, Balter JM, Teng F, Parmar HA, Sahgal A. MR-guided radiation therapy: transformative technology and its role in the central nervous system, Neuro-Oncology, 19, ii16-ii29, 2017.

15) van Sörnsen de Koste JR, Palacios MA, Chen H, Schneiders FL, Bruynzeel AME, Slotman BJ, Haasbeek CJA, Senan S. Changes in gastric anatomy after delivery of breath-hold MR-guided SABR for adrenal metastases. Radiother Oncol. 152:26-29, 2020 doi: 10.1016/j.radonc.2020.07.045.

16) König L, Häfner MF, Katayama S, Koerber SA, Tonndorf-Martini E, Bernhardt D, von Nettelbladt B, Weykamp F, Hoegen P, Klüter S, Susko MS, Debus J, Hörner-Rieber J. Stereotactic body radiotherapy (SBRT) for adrenal metastases of oligometastatic or oligoprogressive tumor patients. Radiat Oncol. 4;15(1):30. 2020 DOI: 10.1186/s13014-020-1480-0

17) Tanaka $O$, Sugiyama $A$, Omatsu $T$, Tawada M, Makita $C$, Matsuo M. Hemostasis radiotherapy for inoperable gastric cancer: A pilot study. Br J Radiol, 93;20190958, 2020

\section{Tables}

Table 1 Organ at risk (OAR) dose constraints applied for three fraction SBRT (54Gy/6fx for CTV) CTV is equal to PTV (Biological effective dose [BED10]) 102Gy Equivalent dose 2Gy (EQD) 85Gy

Radiation dose is limited to OAR. 


\begin{tabular}{|c|c|c|c|c|c|c|}
\hline & D0.1 cc & D5.0 cc & $\mathrm{D} 10.0 \mathrm{cc}$ & V12 Gy & V15 Gy & V21 Gy \\
\hline Stomach & $\leq 22 \mathrm{~Gy}$ & & \multicolumn{2}{|c|}{$\leq 16 \mathrm{~Gy}$} & & \\
\hline Intestine & $\leq 22 \mathrm{~Gy}$ & $\leq 17 \mathrm{~Gy}$ & \multicolumn{2}{|c|}{$\leq 11 \mathrm{~Gy}$} & & \\
\hline Pancreas & $\leq 22 \mathrm{~Gy}$ & & $\leq 12 \mathrm{~Gy}$ & $\leq 50 \%$ & & $\leq 30 \%$ \\
\hline \multicolumn{2}{|l|}{ Liver } & & & & $\leq 50 \%$ & $\leq 30 \%$ \\
\hline \multicolumn{2}{|c|}{ Kidneys (Left) } & \multicolumn{5}{|c|}{$\leq 25 \%$} \\
\hline \multicolumn{2}{|c|}{ Kidneys (Right) } & \multicolumn{3}{|r|}{$\leq 25 \%$} & & \\
\hline \multicolumn{2}{|c|}{ Kidneys (together) } & & & & $\leq 35 \%$ & \\
\hline Spinal cord & $\leq 22 \mathrm{~Gy}$ & & & & & \\
\hline
\end{tabular}

DX (the dose to $\mathrm{X} \%$ of the OARs volume) at $\mathrm{X}$ values of $0.1,5.0$, and $10.0 \mathrm{cc}$.

VX (the percentage of the OARs volume that received more than X Gy)

CTV, clinical target volume; PTV, Planning target volume

Table 2a - Dosing planned by medical physicist 1 


\begin{tabular}{|c|c|c|c|c|}
\hline & & Empty & Full & $\mathrm{p}$-value \\
\hline \multirow[t]{6}{*}{ PTV } & D $0.1 \mathrm{cc}$ & 65.80 & 66.00 & 0.31 \\
\hline & D5 cc & 56.10 & 55.90 & 0.52 \\
\hline & $\mathrm{D} 10 \mathrm{cc}$ & 42.40 & 43.10 & 0.47 \\
\hline & V12 Gy & 99.90 & 99.90 & 0.50 \\
\hline & V15 Gy & 99.60 & 99.80 & 0.22 \\
\hline & V21 Gy & 96.80 & 99.80 & 0.38 \\
\hline \multirow[t]{6}{*}{ Stomach } & $\mathrm{D} 0.1 \mathrm{cc}$ & 19.90 & 20.10 & 0.45 \\
\hline & D5 cc & 13.70 & 15.90 & 0.03 \\
\hline & D10 cc & 12.10 & 14.60 & 0.01 \\
\hline & V12 Gy & 9.20 & 7.80 & 0.69 \\
\hline & V15 Gy & 2.30 & 2.50 & 0.42 \\
\hline & V21 Gy & 0.00 & 0.00 & - \\
\hline \multirow[t]{6}{*}{ Intestine } & D $0.1 \mathrm{cc}$ & 17.80 & 15.30 & 0.20 \\
\hline & D5 cc & 13.40 & 11.00 & 0.17 \\
\hline & D10 cc & 12.20 & 9.60 & 0.15 \\
\hline & V12 Gy & 4.20 & 5.10 & 0.60 \\
\hline & V15 Gy & 1.30 & 2.10 & 0.66 \\
\hline & V21 Gy & 0.00 & 0.00 & - \\
\hline \multirow[t]{6}{*}{ Pancreas } & D $0.1 \mathrm{cc}$ & 45.60 & 38.90 & 0.23 \\
\hline & D5 cc & 26.30 & 19.20 & 0.13 \\
\hline & $\mathrm{D} 10 \mathrm{cc}$ & 20.50 & 13.60 & 0.09 \\
\hline & V12 Gy & 73.40 & 48.40 & 0.06 \\
\hline & V15 Gy & 60.90 & 39.50 & 0.08 \\
\hline & V21 Gy & 37.90 & 22.20 & 0.08 \\
\hline \multirow[t]{6}{*}{ Liver } & $\mathrm{D} 0.1 \mathrm{cc}$ & 15.40 & 13.60 & 0.24 \\
\hline & D5 cc & 11.50 & 10.40 & 0.30 \\
\hline & $\mathrm{D} 10 \mathrm{cc}$ & 10.40 & 9.30 & 0.29 \\
\hline & V12 Gy & 2.00 & 0.80 & 0.12 \\
\hline & V15 Gy & 0.50 & 0.10 & 0.14 \\
\hline & V21 Gy & 0.00 & 0.00 & - \\
\hline \multirow[t]{2}{*}{ Left kidney } & D $0.1 \mathrm{cc}$ & 50.30 & 50.70 & 0.53 \\
\hline & D5 cc & 33.80 & 29.40 & 0.15 \\
\hline
\end{tabular}




\begin{tabular}{|c|c|c|c|c|}
\hline & D10 cc & 25.20 & 20.80 & 0.12 \\
\hline & V12 Gy & 23.80 & 15.60 & 0.01 \\
\hline & V15 Gy & 19.10 & 12.90 & 0.01 \\
\hline & V21 Gy & 12.60 & 8.40 & 0.03 \\
\hline \multirow[t]{6}{*}{ Right Kidney } & D $0.1 \mathrm{cc}$ & 9.80 & 8.40 & 0.13 \\
\hline & D5 cc & 6.90 & 5.90 & 0.21 \\
\hline & D10 cc & 5.50 & 5.10 & 0.38 \\
\hline & V12 Gy & 0.40 & 0.00 & 0.17 \\
\hline & V15 Gy & 0.00 & 0.00 & - \\
\hline & V21 Gy & 0.00 & 0.00 & - \\
\hline \multirow[t]{6}{*}{ Spinal cord } & D $0.1 \mathrm{cc}$ & 14.00 & 14.00 & 0.50 \\
\hline & D5 cc & 7.80 & 7.40 & 0.42 \\
\hline & $\mathrm{D} 10 \mathrm{cc}$ & 2.20 & 1.20 & 0.22 \\
\hline & V12 Gy & 5.90 & 10.40 & 0.92 \\
\hline & V15 Gy & 2.20 & 4.60 & 0.84 \\
\hline & V21 Gy & 0.00 & 0.00 & - \\
\hline
\end{tabular}

DX (the dose to X\% of the Organ at Risks [OARs] volume) at X values of $0.1,5.0,10.0 \mathrm{cc}$.

VX (the percentage of the OARs volume that received more than X Gy)

Table $2 \mathrm{~b}$ - Treatment planned by medical physicist 2 


\begin{tabular}{|c|c|c|c|c|}
\hline & & Empty & Full & $\mathrm{p}$ value \\
\hline \multirow[t]{6}{*}{ PTV } & D $0.1 \mathrm{cc}$ & 66.00 & 66.00 & 0.50 \\
\hline & D5 cc & 56.00 & 53.40 & 0.29 \\
\hline & D10 cc & 44.00 & 40.30 & 0.32 \\
\hline & V12 Gy & 99.90 & 99.90 & 0.50 \\
\hline & V15 Gy & 99.30 & 99.40 & 0.57 \\
\hline & V21 Gy & 97.30 & 97.70 & 0.58 \\
\hline \multirow[t]{6}{*}{ Stomach } & D $0.1 \mathrm{cc}$ & 19.80 & 20.30 & 0.32 \\
\hline & D5 cc & 13.80 & 15.60 & 0.03 \\
\hline & D10 cc & 12.30 & 14.30 & 0.02 \\
\hline & V12 Gy & 7.70 & 6.90 & 0.36 \\
\hline & V15 Gy & 2.50 & 2.20 & 0.37 \\
\hline & V21 Gy & 0.00 & 0.00 & - \\
\hline \multirow[t]{6}{*}{ Intestine } & D $0.1 \mathrm{cc}$ & 17.40 & 15.60 & 0.28 \\
\hline & D5 cc & 13.10 & 11.10 & 0.23 \\
\hline & D10 cc & 11.50 & 9.90 & 0.26 \\
\hline & V12 Gy & 3.40 & 6.20 & 0.20 \\
\hline & V15 Gy & 1.40 & 2.40 & 0.27 \\
\hline & V21 Gy & 0.00 & 0.00 & - \\
\hline \multirow[t]{6}{*}{ Pancreas } & D $0.1 \mathrm{cc}$ & 45.40 & 37.30 & 0.19 \\
\hline & D5 cc & 25.80 & 19.10 & 0.16 \\
\hline & D10 cc & 20.00 & 13.50 & 0.12 \\
\hline & V12 Gy & 70.70 & 44.10 & 0.06 \\
\hline & V15 Gy & 61.10 & 36.80 & 0.06 \\
\hline & V21 Gy & 38.30 & 22.00 & 0.08 \\
\hline \multirow[t]{6}{*}{ Liver } & D $0.1 \mathrm{cc}$ & 16.10 & 15.40 & 0.39 \\
\hline & D5 cc & 12.60 & 11.90 & 0.38 \\
\hline & D10 cc & 11.50 & 10.60 & 0.34 \\
\hline & V12 Gy & 2.60 & 0.70 & 0.06 \\
\hline & V15 Gy & 0.40 & 0.20 & 0.26 \\
\hline & V21 Gy & 0.00 & 0.00 & - \\
\hline \multirow[t]{6}{*}{ Left kidney } & D $0.1 \mathrm{cc}$ & 49.20 & 50.20 & 0.58 \\
\hline & D5 cc & 32.10 & 29.60 & 0.30 \\
\hline & D10 cc & 23.70 & 22.00 & 0.34 \\
\hline & V12 Gy & 22.20 & 15.50 & 0.01 \\
\hline & V15 Gy & 17.70 & 12.90 & 0.03 \\
\hline & V21 Gy & 10.70 & 9.00 & 0.22 \\
\hline \multirow[t]{6}{*}{ Right Kidney } & D $0.1 \mathrm{cc}$ & 10.70 & 11.30 & 0.61 \\
\hline & D5 cc & 8.10 & 9.00 & 0.34 \\
\hline & D10 cc & 6.90 & 8.00 & 0.30 \\
\hline & V12 Gy & 2.60 & 2.30 & 0.55 \\
\hline & V15 Gy & 0.50 & 1.50 & 0.26 \\
\hline & V21 Gy & 0.00 & 0.00 & - \\
\hline Spinal cord & D $0.1 \mathrm{cc}$ & 14.60 & 16.20 & 0.15 \\
\hline
\end{tabular}

Page 13/18 


\begin{tabular}{cccc} 
D5 cc & 7.80 & 6.90 & 0.30 \\
\hline D10 cc & 2.50 & 1.30 & 0.15 \\
\hline V12 Gy & 6.40 & 8.90 & 0.80 \\
\hline V15 Gy & 1.90 & 3.00 & 0.72 \\
\hline V21 Gy & 0.00 & 0.00 & - \\
\hline
\end{tabular}

DX (the dose to X\% of the Organ at Risks [OARs] volume) at X values of 0.1, 5.0, $10.0 \mathrm{cc}$. VX (the percentage of the OARs volume that received more than X Gy)

\section{Figures}

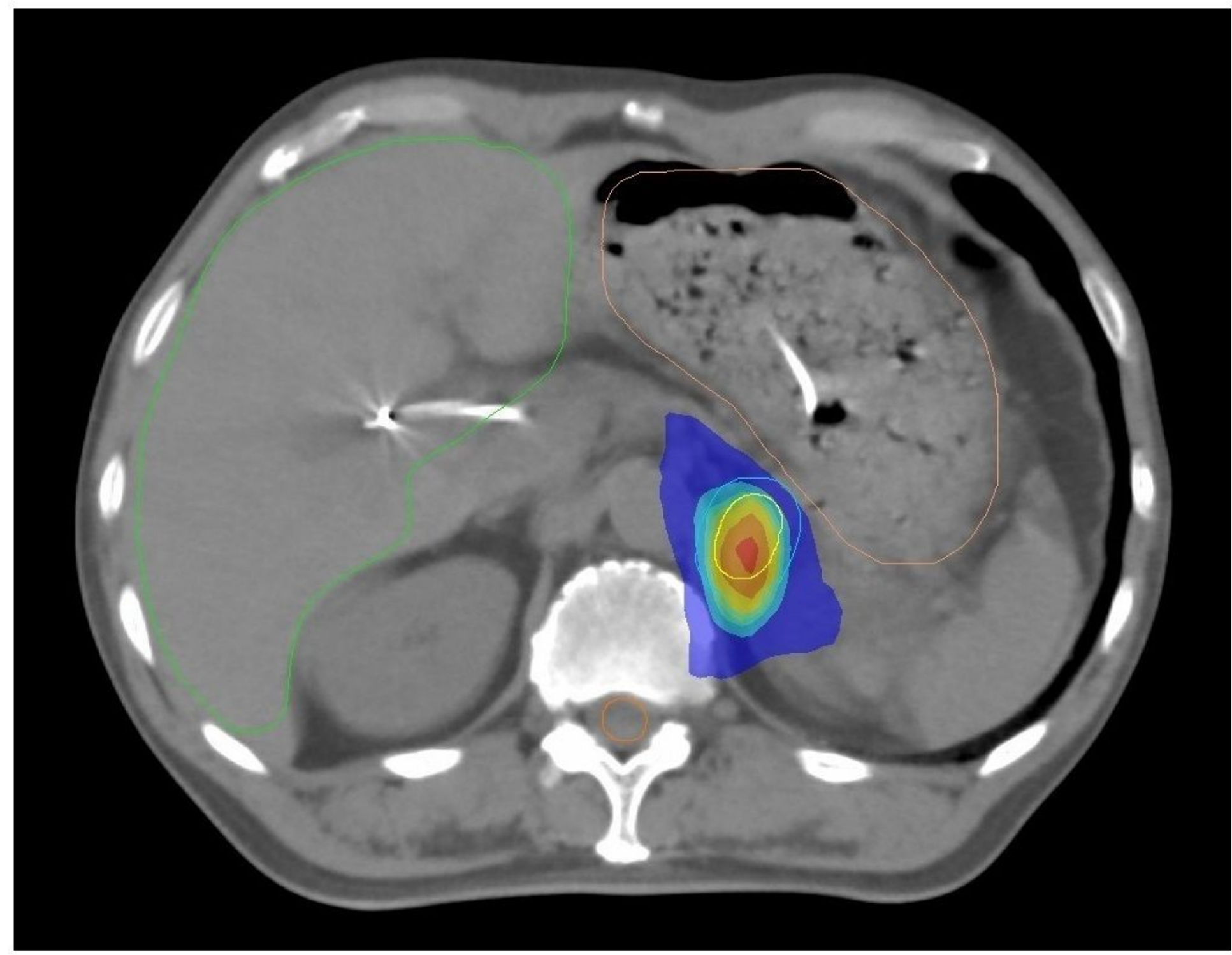

\section{Figure 1}

An example of adrenal SBRT administered in the setting of a large stomach. The pancreas was not shown in this slice. CTV coverage is good Yellow line: adrenal CTV (2 mm margin from GTV) OAR: Liver (green line), Stomach (orange line) 


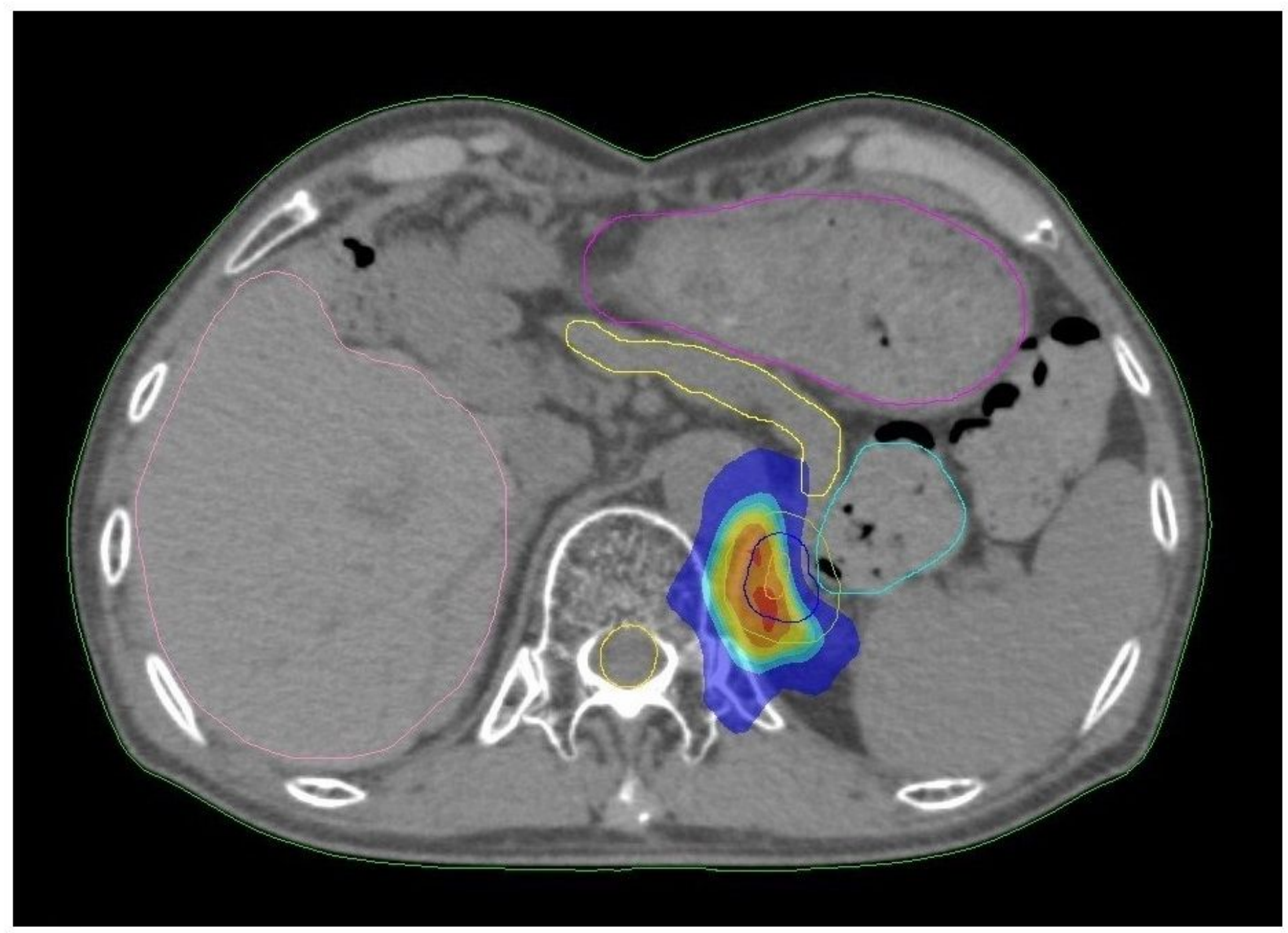

Figure 2

An example of adrenal SBRT in the setting of a large stomach. The intestine is close to the left adrenal ground. CTV coverage is not good due to the intestine. Blue line: adrenal CTV ( $2 \mathrm{~mm}$ margin from GTV) OAR: Liver (pink line), Stomach (purple line), Pancreas (yellow line), and Intestine (light blue line) 


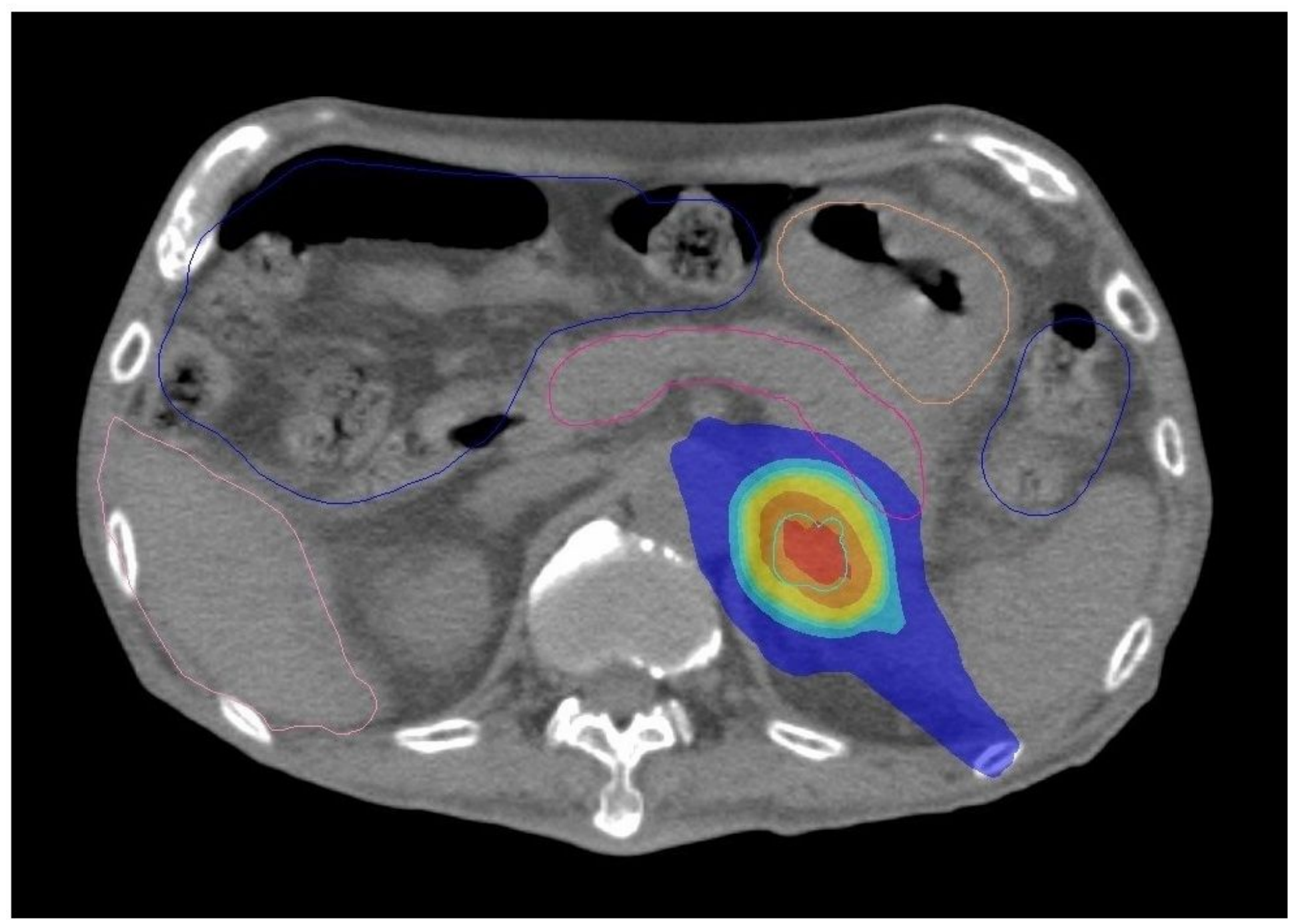

\section{Figure 3}

An example of adrenal SBRT in the setting of a small stomach. The pancreas is close to the left adrenal ground. CTV coverage is good. Light blue line: adrenal CTV (2 mm margin from GTV) OAR: Liver (pink line), Stomach (light orange line), Pancreas (light red line), and Intestine (blue line) 


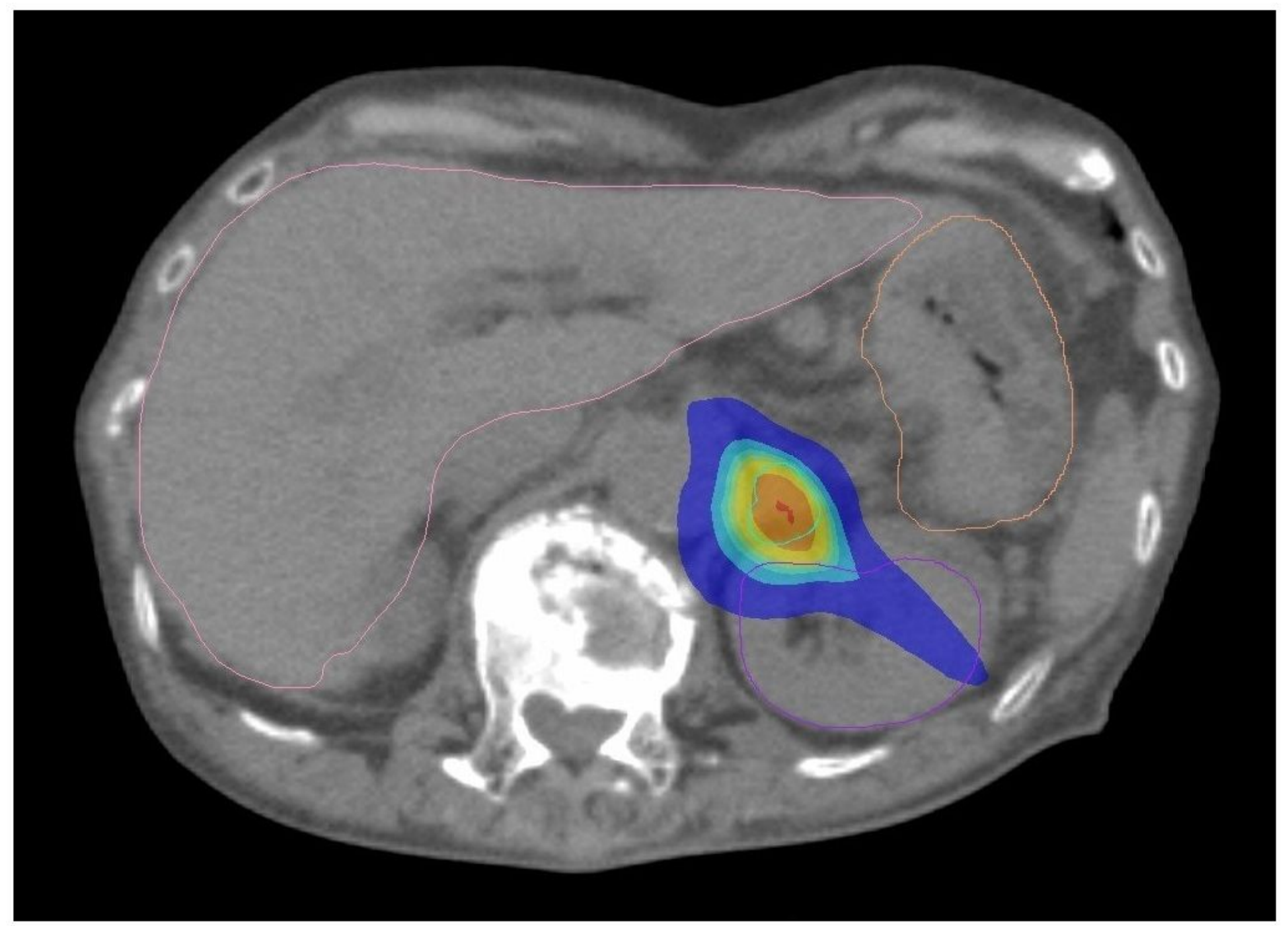

\section{Figure 4}

An example of adrenal SBRT in the setting of a small stomach. The left kidney is close to the left adrenal ground; however, left adrenal CTV coverage is good. Light blue line: adrenal CTV ( $2 \mathrm{~mm}$ margin from GTV) OAR: Liver (pink line), Stomach (light orange line), Pancreas (disappeared in this slide), and Intestine (disappeared in this slide) 


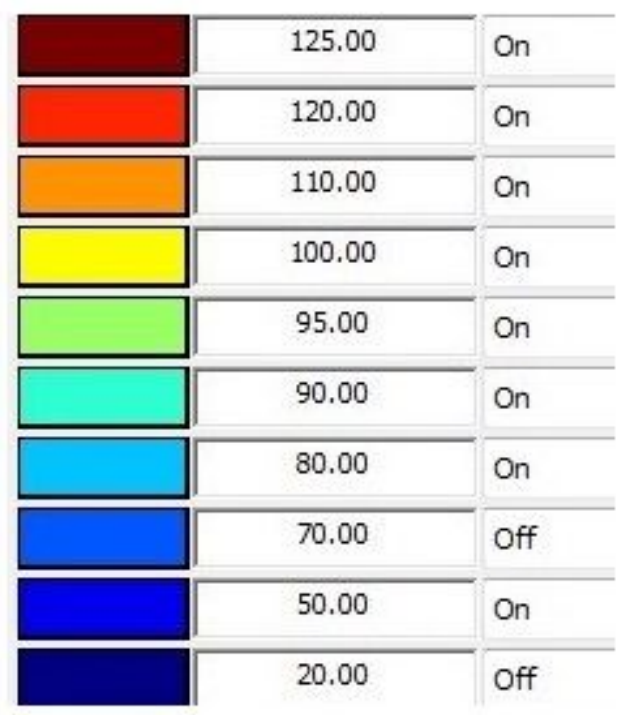

\section{Figure 5}

Color dose wash list (Dose distribution of Figure 1-4) Prescribed dose is $54 \mathrm{~Gy} / 6$ fractions When the area irradiated with 54 Gy is $100 \%$ (yellow), the percentage is expressed by color. 